\title{
An Exploration on the Suitability of Airborne Carbonyl Compounds Analysis in relation to Differences in Instrumentation (GC-MS versus HPLC-UV) and Standard Phases (Gas versus Liquid)
}

\author{
Ki-Hyun Kim, Jan E. Szulejko, Yong-Hyun Kim, and Min-Hee Lee \\ Department of Civil and Environmental Engineering, Hanyang University, 222 Wangsimni-Ro, \\ Seoul 133-791, Republic of Korea
}

Correspondence should be addressed to Ki-Hyun Kim; kkim61@nate.com

Received 2 November 2013; Accepted 23 December 2013; Published 25 February 2014

Academic Editors: R. J. C. Brown, A. D’Annibale, G.-C. Fang, and J. J. Schauer

Copyright (c) $2014 \mathrm{Ki}$-Hyun Kim et al. This is an open access article distributed under the Creative Commons Attribution License, which permits unrestricted use, distribution, and reproduction in any medium, provided the original work is properly cited.

\begin{abstract}
The relative performance figure of merits was investigated for the two most common analytical methods employed for carbonyl compounds (CC), for example, between high performance liquid chromatography (HPLC)-UV detector (with 2,4dinitrophenylhydrazine (DNPH) derivatization) and thermal desorption (TD)-gas chromatography (GC)-mass spectrometry (MS) (without derivatization). To this end, the suitability of each method is assessed by computing the relative recovery (RR) between the gas- and liquid-phase standards containing a suite of CC such as formaldehyde (FA), acetaldehyde (AA), propionaldehyde (PA), butyraldehyde (BA), isovaleraldehyde (IA), and valeraldehyde (VA) along with benzene (B) as a recovery reference for the GC method. The results confirm that a TD-GC-MS is advantageous to attain the maximum recovery for the heavier CCs (i.e., with molecular weights (MW) above BA-MW $\geq 74$ ). On the other hand, the HPLC-UV is favorable for the lighter CCs (like FA and AA) with the least bias. Such compound-specific responses for each platform are validated by relative ordering of CCs as a function of response factor (RF), method detection limit (MDL), and recovery pattern. It is thus desirable to understand the advantages and limitations of each method to attain the CC data with the least experimental bias.
\end{abstract}

\section{Introduction}

The analysis of trace components in a gaseous matrix including ambient air has gained an increasing amount of attention since the 1950's, especially in the field of environmental chemistry, for example, volatile organic compounds (VOC) [1], owing to their potential impacts on human health and to a growing demand for rigorous air quality regulation. Among the wide array of VOC groups, carbonyl compounds (CC) are known to play an important role in secondary organic aerosol (SOA) formation and the associated alteration of climate conditions [2]. Lathière et al. [3] have estimated the total global biogenic volatile organic compound (BVOC) emissions to be $752 \mathrm{Tg}$ C/year (as carbon) for the period 19831995; for instance, the contribution of acetone was estimated as $42 \mathrm{Tg} \mathrm{C} /$ year. The $\mathrm{H}$-abstraction and $\mathrm{NO}_{2}$-addition reactions of aldehydes were studied theoretically to gain insight into the formation of more potent pollutants (e.g., see [4]).

The increasing use of ethanol biofuels such as E85 gasoline is projected to increase acetaldehyde and the associated cancer prevalence in the Los Angeles area in the U.S. [5]. CCs are emitted/produced from cooking activities [6], household furniture [7], biomass fuel combustion [8], sports beverage containers [9], drinking water (as disinfection byproducts (DBP)) [10], and industrial sources [11]. Recently, carbonyl emissions from vehicles running on petroleum or biobased fuels have become a major area in pollution study [12-14] and life-cycle-analysis has been used to determine which fuels (petroleum versus bio) are actually more harmful to the environment, for example, $[15,16]$. Ongoing European 
reforestation projects are projected to increase formaldehyde, acetaldehyde, and acetone biogenic emissions in Europe by $56 \%$ (minimally, globally) and potentially cause European regional climate change [3].

To date, a number of analytical approaches have been proposed and employed for the quantitative analysis of trace CC in ambient air. Among those options, the use of the 2,4-dinitrophenylhydrazine (DNPH) cartridge combined with high performance liquid chromatography (HPLC)-UV detection is often considered the most favorable experimental choice $[6,7,12,13]$. It is however reported that gas chromatographic (GC) analysis combined with pentafluorophenylhydrazine $(\mathrm{PFPH})$ derivatization can yield more reliable data than the HPLC method, when both methods are compared on a parallel basis [17]. Despite such efforts, the efficient detection of CCs in ambient air (and on exhaled breath as potential disease diagnostic biomarkers) still remains a formidable challenge [18].

To facilitate their detection in ambient air, one needs to consider various factors involved in their sampling under field conditions: (a) relative humidity, (b) sampling time, (c) collection (derivatization) efficiency, and (d) $\mathrm{O}_{3}$ denuders that oxidize $\mathrm{NO}$ to $\mathrm{NO}_{2}$. Concerns on the use of $\mathrm{DNPH} /$ sorbent cartridges to sample FA, AA, and acrolein in environmental air over extended periods (up to $24 \mathrm{~h}$ ) have been reviewed with emphasis on $\mathrm{O}_{3}$ and $\mathrm{NO}_{2}$ reactions with DNPH yielding interfering artifacts in the subsequent analysis and sampling time [19]. For example, the AA/FA collection efficiency (CE) from air samples is near 100\% for sampling periods ranging from minutes to a few hours. In contrast, for $24 \mathrm{~h}$ sampling, the reported CE was only $1-62 \%$ and the lower CE is scientifically unexplainable [19]. In contrast, in case of heavier CCs other than AA/FA, large reductions in collection efficiency were also seen from DNPH cartridge method for a normal sampling duration of a few hours [20]. There are a number of reviews that describe a wide array of experimental options to carry our quantitative analysis of CCs [21-23] and VOCs in general [24, 25]. A 2009 review [26] discusses CC detection methods and their limit of detection (LOD). A diverse range of chromophoric and derivatization reagents has been developed and used to facilitate CC detection by HPLC and GC. The use of hydrazine reagents in environmental analysis has been critically reviewed; DNPH is recognized as an international standard [27].

In an effort to characterize the basic methodological approaches available in the CC analysis, we investigated the experimental compatibilities and differences between HPLC and GC methods. To this end, a series of calibration experiments were conducted by both systems using identical standards containing 5 CCs (acetaldehyde (AA), propionaldehyde (PA), butyraldehyde (BA), isovaleraldehyde (IV), and valeraldehyde (VA)) prepared in both gas and liquid phases. The experimental results were then evaluated with respect to the sensitivities or reproducibilities across different carbonyls. In the course of this comparative study, preconcentration of CCs by each system was treated by their basic tools such as cartridge derivatization (HPLC-UV) and sorbent tube trapping (GC). Based on this comparative study, we explore the fundamental properties of each experimental method for CC and discuss their advantages and disadvantages.

\section{Materials and Methods}

2.1. The Significance of Relative Recovery in CC Analysis between Different Standard Phases. The basic information (e.g., molecular formula, molecular weight, density, and chemical structure) of the target carbonyls investigated in this study is briefly summarized in Table 1 . To conduct a calibration-based analysis for the target CCs for a parallel comparison, their working standards (WS) prepared in both gas and liquid phase were analyzed by loading comparable quantities of the target analytes. (See Section 2.2 for the details of standard preparation.) The basic operation conditions for each instrumental setup are summarized in Table 1S (see Table S1 in Supplementary Materials available online at http://dx.doi.org/10.1155/2014/308405).

Note that the analysis of gas standards can be made with similar treatment steps for each method such as derivatization via cartridge sampler (HPLC) and collection via sorbent tube (ST) for thermal desorption (GC). Likewise, the GC-based analysis of liquid standard can be made, similar to real samples, by employing the combined application of sorbent tube collection and thermal desorption treatment. In contrast, it is not the case for the HPLC, as the analysis of the liquid standard can be significantly complicated due to the involvement of reactions leading to their derivatization. Hence, the HPLC analysis could be subject to relatively large biases in the quantitative analysis, if evaluated in terms of compatibility between standard and sample.

Considering all these complicated factors involved in the $\mathrm{CC}$ analysis, the relative recovery (RR) between different standard phases can provide valuable information to assess the analytical reliability of each detection method. The RR of each detection method can be assessed by dividing the difference in response between liquid and gas phase standard by the response of the liquid phase. The computed $R R$ values can thus be used as one of the critical parameters to assess the reliability of the analytical coupling between the standard phases (liquid versus gas) and analytical method (GC versus HPLC).

\subsection{Preparation of CC Standards in Liquid and Gas Phase.} As seen in Tables 2 and 3, standards of two different phases were prepared independently for each method. The gas phase working standards (G-WS) were first prepared separately for each system by diluting gaseous primary standard (G-PS). The G-PS containing the 5 target carbonyls was purchased from Ri Gas Co., (Daejeon, Republic of Korea) containing AA (99.6 ppm), PA (20.1 ppm), BA (18.6 ppm), IV (19.6 ppm), and VA (15.1 ppm). The G-WS for the HPLC calibration was prepared at 5 concentration levels (Table 2(a)), while that for the GC at 3 concentration levels (Table 3(a)). For HPLC analysis, the G-WS of formaldehyde (FA) was also prepared separately by vaporizing formalin solution. FA was then mixed with the G-WS of 5 CCs (from cylinder) to use standard mixture of 6 CCs (Table 2(a)). In contrast, in the case of GC-based analysis, benzene (B) was instead added 
TABLE 1: Basic information of the target carbonyl compounds (CC) selected in this study.

\begin{tabular}{|c|c|c|c|c|c|c|c|c|}
\hline \multirow[t]{2}{*}{ Order } & \multicolumn{2}{|c|}{ Name } & \multirow[t]{2}{*}{ Molecular formula } & \multirow{2}{*}{$\begin{array}{c}\text { MW } \\
\text { (g/mole) }\end{array}$} & \multirow{2}{*}{$\begin{array}{c}\text { Density } \\
(\mathrm{g} / \mathrm{mL})(\mathrm{STP})\end{array}$} & \multicolumn{2}{|c|}{$\begin{array}{l}\text { Retention time } \\
\left(R_{t}\right) \text { (minutes) }\end{array}$} & \multirow[t]{2}{*}{ Structure } \\
\hline & Full name & Short name & & & & HPLC $^{\mathrm{a}}$ & GC-MS & \\
\hline 1 & Formaldehyde & FA & $\mathrm{HCHO}$ & 30.03 & $1.083(\mathrm{aq})$ & 3.2 & - & \\
\hline 2 & Acetaldehyde & AA & $\mathrm{CH}_{3} \mathrm{CHO}$ & 44.05 & $0.788(1)$ & 3.8 & 5.6 & \\
\hline 3 & Propionaldehyde & PA & $\mathrm{CH}_{3} \mathrm{CH}_{2} \mathrm{CHO}$ & 58.08 & $0.798(1)$ & 4.8 & 7.7 & \\
\hline 4 & Butyraldehyde & BA & $\mathrm{CH}_{3} \mathrm{CH}_{2} \mathrm{HCH}_{2} \mathrm{CHO}$ & 72.11 & $0.817(\mathrm{l})$ & 6.2 & 8.2 & \\
\hline 5 & Isovaleradehyde & IA & $\left(\mathrm{CH}_{3}\right)_{2} \mathrm{CHCH}_{2} \mathrm{CHO}$ & 86.13 & $0.8209(1)$ & 7.9 & 8.6 & \\
\hline 6 & Valeraldehyde & VA & $\mathrm{CH}_{3}\left(\mathrm{CH}_{2}\right)_{3} \mathrm{CHO}$ & 86.13 & $0.8095(1)$ & 8.3 & 10 & \\
\hline
\end{tabular}

${ }^{\mathrm{a}}$ Retention time based on the mobile phase composition of ACN : water $(70: 30)$ used in this study.

into the G-WS of 5 CCs as a reference compound due to its stability and good recovery. As the GC-MS conditions were not feasible to quantitate the low molecular weight compound like FA, GC-based analysis was only confined to five CCs from the cylinder (Table 3(a)). Hence, the selection of the target components by the two systems is distinguished in that FA and benzene are measured in addition to the five main CC targets for the HPLC and GC-MS, respectively.

The liquid phase WS (L-WS) for each system was also prepared independently for the comparative calibration by each system. As shown in Table 2(b), the L-WS for the HPLCbased analysis was prepared at 5 different concentration levels using the standard commercially available carbonyldinitrophenylhydrazine (DNPH) mix (Supelco, USA). In contrast, the L-WS for the GC-based analysis was prepared gravimetrically as shown in Table $3(\mathrm{~b})$. These L-WS were made to cover three different concentration levels using the primary grade chemicals purchased at the purity of $\geq 99 \%$ (Sigma-Aldrich, USA). They were prepared independently from those of HPLC to avoid interfering effects of DNPH in the detection stage of GC-MS. (Note that the L-WS for HPLC analysis is made on the basis of DNPH derivatization). In the case of the GC-based analysis, the L-WS was also made to contain benzene as a reference compound along with the five target CCs for direct comparison with gas-phase standard.

2.3. Carbonyl Analysis by HPLC Method. To assess the relative recovery of HPLC-based calibration data between the two standard phases, the calibration results were derived from both G-WS and L-WS in a comparable manner. In the case of G-WS, each of all five standard samples (five concentrations, Table 3(a)) was pulled into the cartridge to induce derivatization with DNPH. The collection of CCs from G-WS was made by the cartridges prepacked with chromatographic grade silica (60-100 mesh) and coated with 2,4-DNPH (1 mg/cartridge) (Supelco Inc., PA, USA). All standards were collected into the cartridges at a fixed flow rate of $1 \mathrm{~L} \mathrm{~min}^{-1}$ for 8 minutes and regulated by a vacuum pump with an adjustable flow controller (MP- $\sum 300$, SIBATA, Tokyo, Japan). Teflon tubing was used to connect the Tedlar bag, DNPH cartridge, and flow controller. After each sampling, the cartridges were capped and wrapped in pouches (Supelco Inc., PA, USA). The pouches were stored in desiccators until the carbonyl analysis was performed (e.g., within 2 hours).

The carbonyl-hydrazones were analyzed by HPLC (Lab Alliance 500) equipped with a UV detector and dsCHROM software for peak integration. To initiate the HPLC-based analysis of G-WS, the cartridges were eluted slowly with acetonitrile into a $5 \mathrm{~mL}$ capacity borosilicate glass volumetric flask. The eluate was injected into the HPLC system equipped with a $20 \mu \mathrm{L}$ sample loop. Different carbonyl-hydrazones were separated on a Hichrom $250 \times 4.6 \mathrm{~mm}$ ODS (octadecylsilane), $5 \mu \mathrm{m}$ reverse phase $\mathrm{C}_{18}$ column using a mobile phase of acetonitrile + water $(6.5: 3.5$ by volume $)$ at a flow rate of $1.5 \mathrm{~mL} \mathrm{~min}^{-1}$. The final calibration of the gaseous CCs was performed by injecting $20 \mu \mathrm{L}$ of each eluate taken from five different G-WS. In case of L-WS, samples of DNPH mix standard prepared at five concentration levels (Table 2(b)) were analyzed by injecting $20 \mu \mathrm{L}$ of each into the HPLC.

2.4. Carbonyl Analysis by TD/GC/MS Method. To perform the comparison between different analytical approaches in CC analysis, the performance of the TD/GC/MS method was also investigated between the gas- and liquid-phase standards (Table 3). To conduct the GC-based calibration, standards prepared in both phases were treated in an identical manner; each of them was initially loaded on the sorbent tube and 
TABLE 2: Comparison of HPLC-based calibration analysis of CC between gas- and liquid-phase standards.

(a) Calibration of gas-phase CC standard by HPLC/DNPH cartridge method

\begin{tabular}{|c|c|c|c|c|c|c|}
\hline Order & FA & AA & PA & BA & IA & VA \\
\hline \multicolumn{7}{|c|}{ Concentration of CC standard (ppb) for 5-point analysis } \\
\hline 1 & 96.1 & 65.1 & 13.1 & 12.2 & 12.8 & 9.90 \\
\hline 2 & 194 & 132 & 26.6 & 24.6 & 25.9 & 20.0 \\
\hline 3 & 387 & 264 & 53.0 & 49.2 & 51.9 & 40.0 \\
\hline 4 & 773 & 527 & 106 & 98.0 & 104 & 80.0 \\
\hline 5 & 1,559 & 1,058 & 213 & 198 & 208 & 160 \\
\hline \multicolumn{7}{|c|}{ Calculated mass (ng) of CC injected into HPLC ${ }^{\mathrm{a}}$ assuming no losses in derivatization and extraction } \\
\hline 1 & 3.79 & 3.79 & 1.01 & 1.16 & 1.46 & 1.12 \\
\hline 2 & 7.58 & 7.58 & 2.02 & 2.31 & 2.91 & 2.24 \\
\hline 3 & 15.2 & 15.2 & 4.03 & 4.63 & 5.83 & 4.49 \\
\hline 4 & 30.3 & 30.3 & 8.06 & 9.26 & 11.7 & 8.98 \\
\hline 5 & 60.6 & 60.6 & 16.1 & 18.5 & 23.3 & 18.0 \\
\hline \multicolumn{7}{|c|}{ Peak area } \\
\hline 1 & $1,430,395$ & 882,104 & 114,854 & 164,484 & 74,796 & 80,079 \\
\hline 2 & $2,754,983$ & $1,733,480$ & 222,048 & 297,748 & 146,260 & 177,013 \\
\hline 3 & $5,650,140$ & $3,472,465$ & 577,168 & 640,128 & 375,504 & 449,499 \\
\hline 4 & $11,240,906$ & $6,552,152$ & $1,007,331$ & $1,059,943$ & 728,865 & 894,010 \\
\hline 5 & $22,526,453$ & $12,305,560$ & $1,718,513$ & $1,742,954$ & $1,454,395$ & $1,660,223$ \\
\hline
\end{tabular}

${ }^{\mathrm{a}}$ For each calibration point, $8 \mathrm{~L}$ of gaseous CC standard is sampled by the cartridge and these CCs are extracted by $5 \mathrm{~mL}$ acetonitrile. As $20 \mu \mathrm{L}$ of extract is injected into HPLC, the actual mass (ng) of CC loaded onto HPLC is computed as the total quantity of each CC contained in $20 \mu \mathrm{L}$ extract.

(b) HPLC calibration results for liquid-phase CC standard

\begin{tabular}{lccccccc}
\hline $\begin{array}{l}\text { Concentration } \\
\left(\mathrm{ng} \mu \mathrm{L}^{-1}\right)\end{array}$ & $\begin{array}{c}\text { Loading } \\
\text { mass }(\mathrm{ng})^{\mathrm{a}}\end{array}$ & FA & AA & PA & BA & IA & VA \\
\hline 0.15 & 3 & $1,150,435$ & 886,294 & 606,404 & 502,987 & 259,055 & 358,175 \\
0.30 & 6 & $2,137,986$ & $1,584,941$ & $1,247,462$ & 940,570 & 555,295 & 666,852 \\
0.60 & 12 & $4,243,306$ & $3,183,149$ & $2,388,591$ & $1,987,032$ & $1,391,763$ & $1,425,906$ \\
1.20 & 24 & $7,763,079$ & $5,875,879$ & $4,646,142$ & $3,691,885$ & $2,922,397$ & $2,786,051$ \\
2.40 & 48 & $15,043,330$ & $11,578,866$ & $9,202,951$ & $7,322,176$ & $6,099,023$ & $5,976,525$ \\
\hline
\end{tabular}

${ }^{a}$ Aldehyde/ketone-DNPH mix (Supelco): liquid phase standard is prepared to have equal mass for all target compounds per unit volume.

subject to thermal desorption in a consistent manner. The sorbent tube was packed with $300 \mathrm{mg}$ of the Carbopack X sorbent (mesh 60/80). Once the sorbent tube was loaded with WS, it was subjected to the adsorption/desorption cycle inside the TD system. The cold (or cryofocusing) trap in the TD system was packed with two sorbent materials of Tenax TA and Carbopack B at equivolume ratio. The selection of sorbents used in the present work was based on experiments done in our laboratory $[28,29]$. The adsorption and desorption of the analytes in the TD system were carried out at $5^{\circ} \mathrm{C}(5 \mathrm{~min})$ and $320^{\circ} \mathrm{C}(20 \mathrm{~min})$, respectively. The carbonyl compounds were then separated on a CP-Wax column (diameter: $0.25 \mathrm{~mm}$, length: $60 \mathrm{~m}$, and thickness: $0.25 \mu \mathrm{m}$ ) with a split ratio of $1: 5$. The column temperature was ramped at $10^{\circ} \mathrm{C} \mathrm{min}^{-1}$ from an initial temperature of $40^{\circ} \mathrm{C}$ to the final temperature of $180^{\circ} \mathrm{C}$. Helium (>99.999\%) was used as a carrier gas at a flow rate of $1 \mathrm{~mL} \mathrm{~min}^{-1}$. The detection of carbonyl compounds was made by an MS interfaced to the $\mathrm{GC}, 70 \mathrm{eV}$ EI ionization, and $200^{\circ} \mathrm{C}$ ion source temperature.
Total ion chromatograms (TIC) mode was used over a mass range of $\mathrm{m} / \mathrm{z} 35$ to 250 .

In the case of G-WS, the inlet of the sorbent tube was connected to a standard storage container (polyester aluminum: PEA) filled with gas-phase WS via Teflon tubing, and the outlet of the sorbent tube was then connected to a vacuum pump (MP- $\Sigma 30$, Shibata, Japan). The transfer of GWS into the sorbent tube was initiated at a fixed flow rate of $100 \mathrm{~mL} \mathrm{~min}^{-1}$ for $1 \mathrm{~min}$. The sorbent tube loaded with G-WS was then thermally desorbed to derive calibration curves for each target compound.

The same sorbent tube was also used to calibrate the LWS. The inlet and outlet of the sorbent tube were connected with a PEA container filled with ultra-pure nitrogen and the vacuum pump, respectively. $1 \mu \mathrm{L}$ of L-WS prepared at three concentration levels was spiked on the inlet of the sorbent tube using a $10 \mu \mathrm{L}$ liquid syringe. A purge of ultrapure nitrogen was applied to the ST prior to TD analysis to reduce the solvent effect of liquid standard at a flow rate 
TABLE 3: Comparison of TD/GC/MS-based calibration analysis of CC using both standard phases.

(a) GC-based calibration of gas-phase CC standard

\begin{tabular}{|c|c|c|c|c|c|c|}
\hline Order & $\mathrm{B}^{\mathrm{a}}$ & AA & $\mathrm{PA}$ & $\mathrm{BA}$ & IA & VA \\
\hline \multicolumn{7}{|c|}{ Concentration (ppb) } \\
\hline 1 & 40.0 & 199 & 40.2 & 37.2 & 39.2 & 30.2 \\
\hline 2 & 80.0 & 398 & 80.4 & 74.4 & 78.4 & 60.4 \\
\hline 3 & 160 & 797 & 161 & 149 & 157 & 121 \\
\hline \multicolumn{7}{|c|}{ The actual mass (ng) of CC injected into TD/GC ${ }^{b}$} \\
\hline 1 & 12.8 & 35.9 & 9.5 & 11.0 & 13.8 & 10.6 \\
\hline 2 & 25.6 & 71.8 & 19.1 & 21.9 & 27.6 & 21.3 \\
\hline 3 & 51.1 & 144 & 38.2 & 43.9 & 55.2 & 42.6 \\
\hline \multicolumn{7}{|c|}{ Peak area } \\
\hline 1 & $1,835,850$ & 66,043 & 114,313 & 615,231 & $1,105,826$ & 808,071 \\
\hline 2 & $3,516,064$ & 101,214 & 218,491 & $1,198,036$ & $2,257,147$ & $1,578,254$ \\
\hline 3 & $6,794,287$ & 103,975 & 431,169 & $2,428,141$ & $4,484,753$ & $3,306,639$ \\
\hline
\end{tabular}

${ }^{\mathrm{a}}$ Because of limitation in the analysis of FA, benzene (B) was analyzed in place of FA.

${ }^{\mathrm{b}}$ Flow rate $=100 \mathrm{~mL} \mathrm{~min}^{-1}$, loading time $=1 \mathrm{~min}$, and loading volume $=100 \mathrm{~mL}$.

(b) GC-based calibration of liquid-phase CC standard

\begin{tabular}{|c|c|c|c|c|c|c|}
\hline Order & $\mathrm{B}$ & $\mathrm{AA}$ & $\mathrm{PA}$ & $\mathrm{BA}$ & IA & VA \\
\hline \multicolumn{7}{|c|}{ Concentration $\left(\mathrm{ng} \mu \mathrm{L}^{-1}\right)$ of CC injected into $\mathrm{GC}^{\mathrm{c}}$} \\
\hline 1 & 10.1 & 27.2 & 9.03 & 9.30 & 9.02 & 9.17 \\
\hline 2 & 20.2 & 54.4 & 18.1 & 18.6 & 18.0 & 18.3 \\
\hline 3 & 40.4 & 109 & 36.1 & 37.2 & 36.1 & 36.7 \\
\hline \multicolumn{7}{|c|}{ Peak area } \\
\hline 1 & $1,502,193$ & 76,511 & 36,105 & 425,976 & 641,460 & 612,205 \\
\hline 2 & $2,784,977$ & 172,068 & 113,520 & 954,543 & $1,503,596$ & $1,489,447$ \\
\hline 3 & $5,361,966$ & 351,382 & 234,837 & $1,957,700$ & $2,992,220$ & $3,077,189$ \\
\hline
\end{tabular}

${ }^{\mathrm{c}} \mathrm{GC}$ injection volume: $1 \mu \mathrm{L}$.

of $200 \mathrm{~mL} \mathrm{~min}^{-1}$ for $5 \mathrm{~min}$ [30]. The purge procedure can suppress or decrease solvent (methanol) interference with target components contained in L-WS. It can eventually help minimize any possible bias in the sample adsorption stage.

\section{Results and Discussion}

3.1. Comparison of the CC Calibration Trends between the Two Methods. The basic properties of each detection method used for the quantitation of CCs can be assessed by comparing the relative calibration patterns of each method. The raw calibration data for the HPLC and GC method are presented in Tables 2 and 3, respectively. As shown in Table 2, the HPLC-based analysis was tested for five odorous CCs plus FA across five calibration points. In comparison, the GC/MSbased analysis for five CCs and benzene was made as three point calibrations for both liquid and gas phase standards (Table 3). Because of the excellent linearity with high $R^{2}$ values, all GC-MS analyses were confined to three point calibration for the sake of simplicity. The results of the HPLC calibration experiments derived by both standard phases are plotted in Figure 1. Likewise, the comparable data sets derived by GC/MS are also depicted in Figure $1 \mathrm{~S}$.

As shown in Table 4, the results of these calibration experiments can be summarized in terms of response factor
(RF) and coefficient of determination $\left(R^{2}\right)$. These results are also presented to allow comparison between the two different experimental methods and between the two standard phases. The results of our experiments show that the calibration data obtained by the HPLC generally maintained higher $R^{2}$ values for all CCs, although results of PA and BA were slightly lower with 0.98 and 0.96 , respectively. Likewise, the GC-based experiments also yielded fairly good calibration results with an exception of AA prepared in gas phase. In our previous study, the TD-GC analysis of the lighter CCs like AA was found to suffer from limited linearity depending on the sorbent material type used in the cryofocusing stage [28, 29].

Although the calibration results derived by both systems cannot be compared in absolute terms, their relative patterns between different compounds can be assessed very meaningfully. According to this compilation, the relative ordering in RF values of the HPLC method decreases monotonically (as 1/MW: L-WS $R^{2}=0.986$ and G-WS $R^{2}$ $=0.967)$ with increasing molecular weight $(\mathrm{MW})$, regardless of standard phase. As expected, the $360 \mathrm{~nm}$ UV molar extinction coefficients are very similar across all analyzed carbonyl-dinitrophenylhydrazine derivatives [31]. The corresponding molar MDL's for HPLC/UV analysis of the LWS aldehyde/ketone-DNPH mix are $\sim 0.80 \pm 0.02 \mathrm{pMol}$ (see Table 5). The present work's MDL results are inconsistent with 

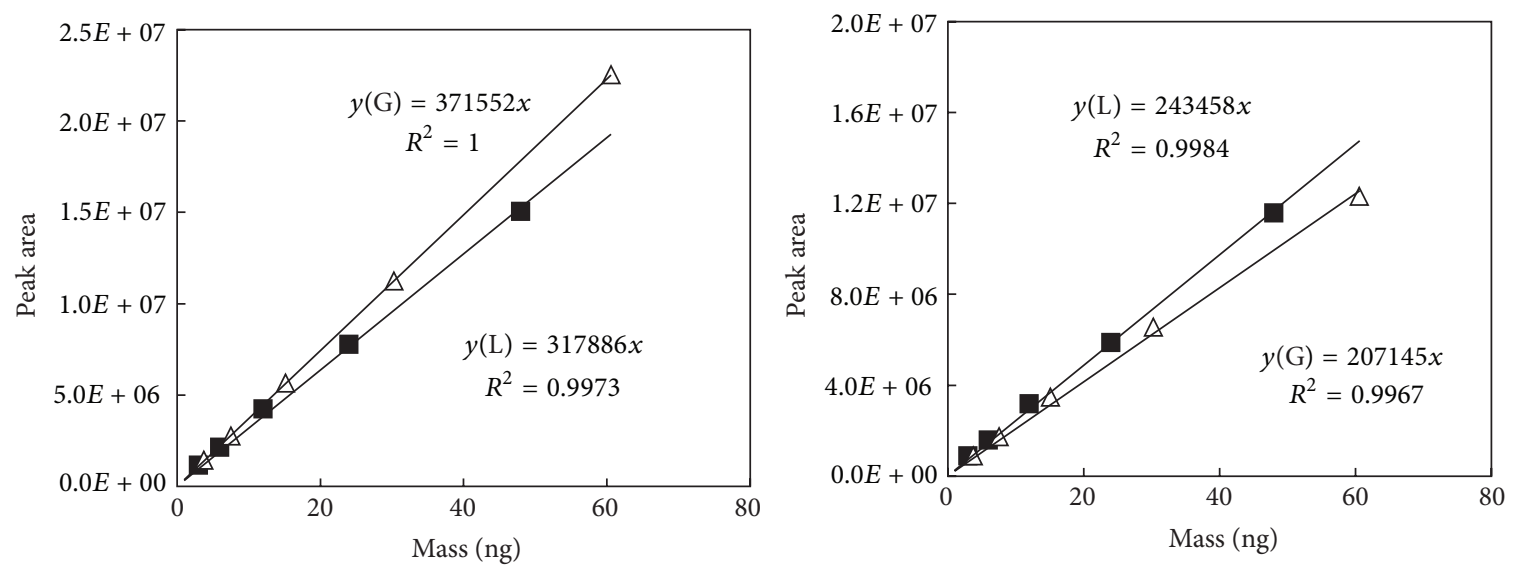

- $\mathrm{FA}(\mathrm{L})$

- $\mathrm{AA}(\mathrm{L})$

$\triangle \mathrm{FA}(\mathrm{G})$
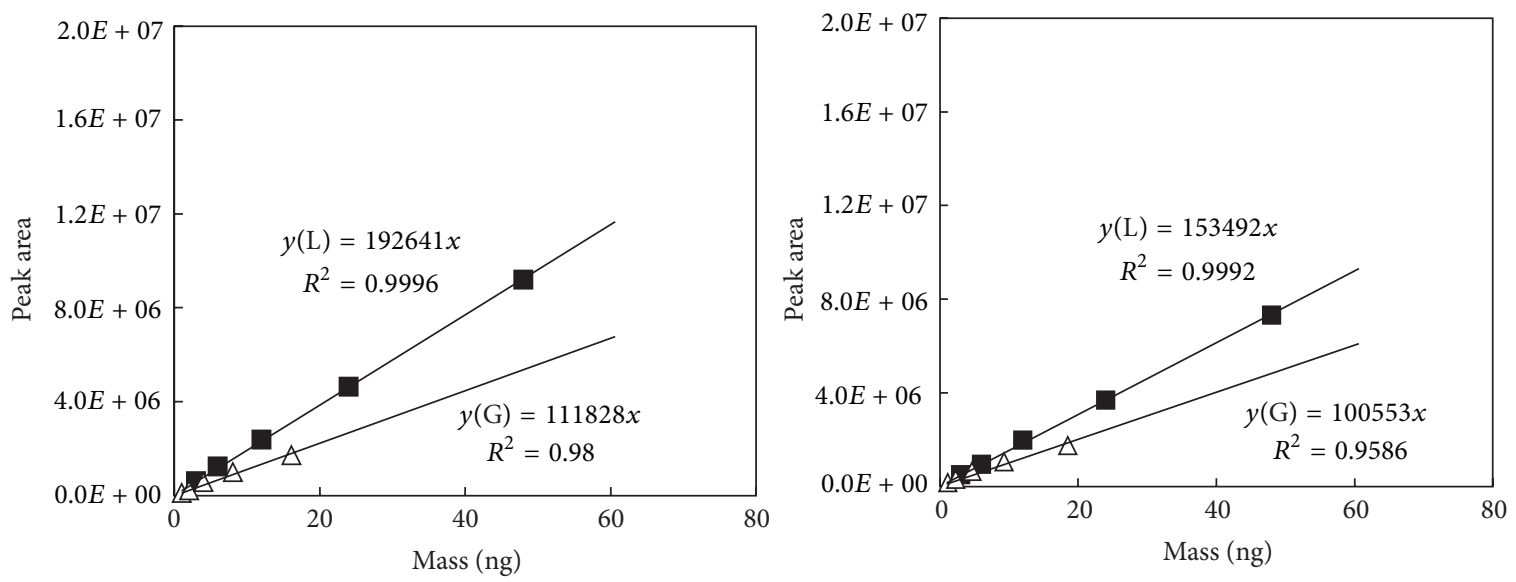

- $\mathrm{PA}(\mathrm{L})$

$\triangle \mathrm{PA}(\mathrm{G})$

$\mathrm{BA}(\mathrm{L})$

$\triangle \mathrm{BA}(\mathrm{G})$
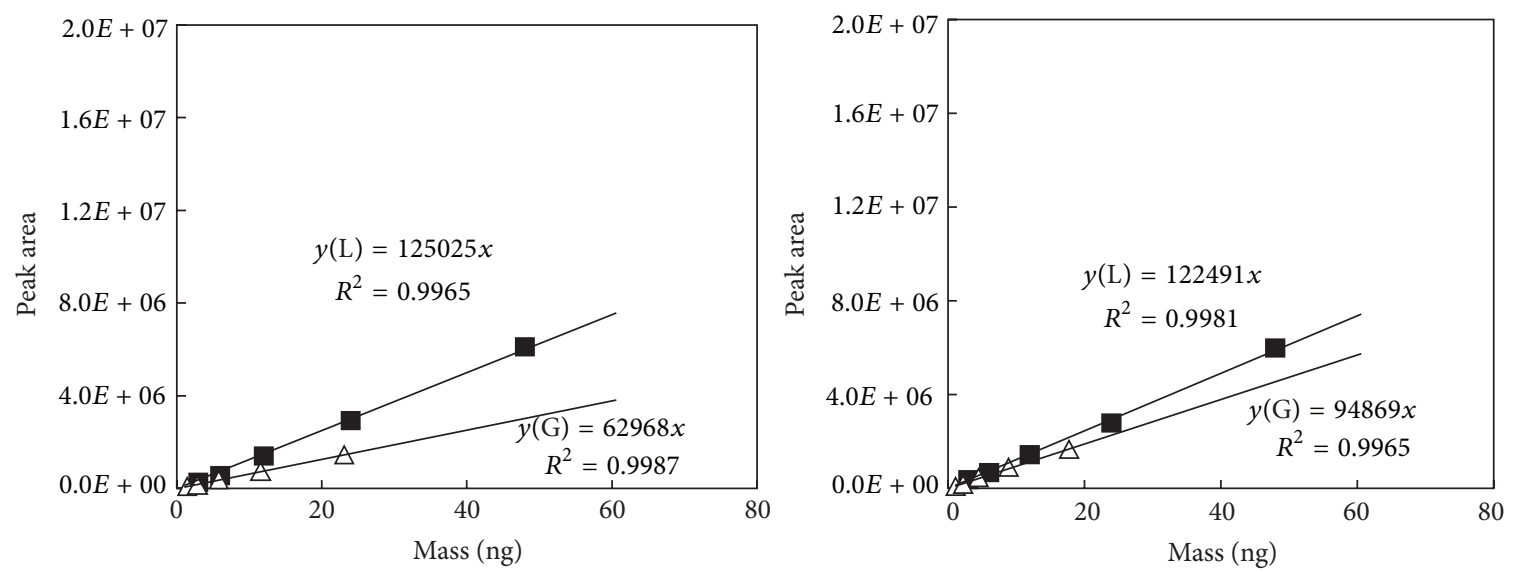

- IA(L)

- $\mathrm{VA}(\mathrm{L})$

$\Delta \mathrm{IA}(\mathrm{G})$

$\triangle \mathrm{VA}(\mathrm{G})$

FIGURE 1: Comparison of HPLC-based calibration curves of CCs between liquid- (L) and gas-phase (G) standards. 
TABLE 4: Comparison of relative recovery of each CC between different standard phases for a given method.

\begin{tabular}{|c|c|c|c|c|c|c|c|}
\hline Method & Type & FA/B & AA & $\mathrm{PA}$ & $\mathrm{BA}$ & IA & VA \\
\hline \multirow{7}{*}{ HPLC-UV } & $\mathrm{RF}(\mathrm{L})$ & 317,886 & 243,458 & 192,641 & 153,492 & 125,025 & 122,491 \\
\hline & $\mathrm{RF}(\mathrm{L}) * \mathrm{MW}^{\mathrm{a}}$ & 9.6 & 10.7 & 11.2 & 11.0 & 10.8 & 10.5 \\
\hline & $R^{2}(\mathrm{~L})$ & 0.997 & 0.998 & 1.000 & 0.999 & 0.997 & 0.998 \\
\hline & $\mathrm{RF}(\mathrm{G})$ & 371,552 & 207,145 & 111,828 & 100,553 & 62,964 & 94,871 \\
\hline & $\mathrm{RF}(\mathrm{G}) * \mathrm{MW}^{\mathrm{a}}$ & 11.1 & 9.1 & 6.3 & 7.3 & 5.4 & 8.2 \\
\hline & $R^{2}(\mathrm{G})$ & 1.000 & 0.997 & 0.980 & 0.959 & 0.999 & 0.997 \\
\hline & $\mathrm{PD}$ & -17 & 15 & 42 & 34 & 50 & 23 \\
\hline \multirow{8}{*}{ GC-EI-MS $^{b}$} & $\operatorname{RRF}\left(\sigma_{r}\right)^{c}$ & 100 & 66 & 67 & 68 & 69 & 69 \\
\hline & $\mathrm{RF}(\mathrm{L})$ & 134,475 & 3,197 & 6,341 & 52,065 & 82,455 & 82,598 \\
\hline & Rel RF(L) & 100 & 2.4 & 4.7 & 39 & 61 & 61 \\
\hline & $R^{2}(\mathrm{~L})$ & 0.9961 & 0.9968 & 0.976 & 0.9967 & 0.9961 & 0.9923 \\
\hline & $\mathrm{RF}(\mathrm{G})$ & 134,336 & 747 & 10,874 & 55,232 & 81,249 & 76,956 \\
\hline & $R^{2}(\mathrm{G})$ & 0.9979 & 0.906 & 0.9953 & 0.9998 & 0.9999 & 0.9986 \\
\hline & Rel RF(G) & 100 & 0.56 & 8.1 & 41 & 60 & 57 \\
\hline & $\mathrm{PD}$ & 0.10 & 76.65 & -71.49 & -6.08 & 1.46 & 6.83 \\
\hline
\end{tabular}

${ }^{a}$ Divided by 1,000,0000 to give small numbers for convenience-essentially molar RF figure of merit; ${ }^{b}$ because of limitation in the analysis of FA, benzene (B) is analyzed in replacement of FA, and ${ }^{\mathrm{c}} \mathrm{RRF}\left(\sigma_{r}\right)$ is based on estimated EI total ionization cross sections (see text for details).

TABLE 5: Comparison of basic QA parameters in CC analysis between different standard phases using both HPLC and GC methods.

(a) Precision (relative standard error (RSE): in \%)

\begin{tabular}{lccccccc}
\hline & & FA/B & AA & PA & BA & IA & VA \\
\hline \multirow{2}{*}{ HPLC } & Gas $^{\mathrm{a}}$ & $1.81(\mathrm{FA})$ & 1.97 & 2.33 & 2.26 & 1.76 & 1.92 \\
& Liquid $^{\mathrm{b}}$ & $0.46(\mathrm{FA})$ & 0.80 & 0.92 & 0.67 & 0.70 & 0.78 \\
\hline \multirow{2}{*}{ GC-MS } & Gas $^{\mathrm{c}}$ & $0.23(\mathrm{~B})$ & 0.24 & 0.35 & 0.55 & 0.03 & 0.49 \\
& Liquid $^{\mathrm{d}}$ & $3.46(\mathrm{~B})$ & 1.14 & 4.32 & 2.61 & 3.14 & 3.34 \\
\hline
\end{tabular}

${ }^{\mathrm{a}}$ Loading volume $=8 \mathrm{~L}\left(8 \mathrm{~min}\right.$ at a flow rate $\left.=1 \mathrm{~L} \mathrm{~min}^{-1}\right)$ of 121 (VA) to $1171 \mathrm{ppb}$ standard (FA); ${ }^{\mathrm{b}}$ injection amount $=20 \mu \mathrm{L}$ of $0.6 \mathrm{ng} \mu \mathrm{L}^{-1}(12 \mathrm{ng}) ;{ }^{\mathrm{c}}$ loading volume $=100 \mathrm{~mL}\left(1 \mathrm{~min}\right.$ at flow rate $\left.=100 \mathrm{~mL} \mathrm{~min}^{-1}\right)$ of $38(\mathrm{VA})$ to $250 \mathrm{ppb}$ standard (AA); and ${ }^{\mathrm{d}} 1 \mu \mathrm{L}$ injection of 18 (VA) to $54 \mathrm{ng} \mu \mathrm{L}^{-1}$ (AA) liquid standard.

(b) Detection limits

\begin{tabular}{|c|c|c|c|c|c|c|c|c|}
\hline & & Units $^{\mathrm{a}}$ & $\mathrm{FA} / \mathrm{B}$ & $\mathrm{AA}$ & $\mathrm{PA}$ & $\mathrm{BA}$ & IA & VA \\
\hline \multirow{5}{*}{ HPLC } & \multirow{2}{*}{$\mathrm{Gas}^{\mathrm{b}}$} & pg & 21.0 (FA) & 37.7 & 69.8 & 77.6 & 123.9 & 82.2 \\
\hline & & $\mathrm{ppb}$ & 0.53 (FA) & 0.65 & 0.92 & 0.82 & 1.10 & 0.73 \\
\hline & \multirow{3}{*}{ Liquid $^{c}$} & pg & 27.6 (FA) & 36.0 & 45.5 & 57.2 & 70.2 & 71.6 \\
\hline & & $\mathrm{ppb}$ & $0.70(\mathrm{FA})$ & 0.63 & 0.60 & 0.61 & 0.62 & 0.64 \\
\hline & & pMol & $0.92(\mathrm{FA})$ & 0.82 & 0.78 & 0.79 & 0.83 & 0.82 \\
\hline \multirow{4}{*}{ GC-MS } & \multirow{2}{*}{ Gas $^{\mathrm{d}}$} & ng & 0.07 (B) & 9.68 & 0.81 & 0.16 & 0.11 & 0.11 \\
\hline & & $\mathrm{ppb}$ & $0.02(\mathrm{~B})$ & 5.37 & 0.34 & 0.05 & 0.03 & 0.03 \\
\hline & \multirow{2}{*}{ Liquid $^{\mathrm{e}}$} & ng & 0.07 (B) & 2.97 & 1.50 & 0.18 & 0.12 & 0.12 \\
\hline & & $\mathrm{ppb}$ & $0.02(\mathrm{~B})$ & 1.65 & 0.63 & 0.06 & 0.03 & 0.03 \\
\hline
\end{tabular}

${ }^{\mathrm{a}}$ To calculate concentration in ppb, the total sample volumes are assumed as 8 (HPLC) and $1 \mathrm{~L}$ (GC-MS); ${ }^{\mathrm{b}}$ loading volume $=8 \mathrm{~L}$ ( $8 \mathrm{~min}$ at a flow rate $\left.=1 \mathrm{~L} \mathrm{~min}{ }^{-1}\right)$ of $2.7(\mathrm{VA})$ to $26 \mathrm{ppb}$ standard (FA); ${ }^{\mathrm{c}}$ injection amount $=0.5 \mathrm{ng}\left(20 \mu \mathrm{L}\right.$ of $\left.0.025 \mathrm{ng} \mu \mathrm{L}^{-1}\right) ;{ }^{\mathrm{d}}$ loading volume $=50 \mathrm{~mL}(1 \mathrm{~min}$ at flow rate $=$ $50 \mathrm{~mL} \mathrm{~min}^{-1}$ ) of 0.76 (VA) to $4.98 \mathrm{ppb}$ standard (AA); and ${ }^{\mathrm{e}} 1 \mu \mathrm{L}$ injection of 0.29 (IA) to $0.87 \mathrm{ng} \mu \mathrm{L}^{-1}$ (AA) liquid standard.

the almost constant MDL $\left(0.04-0.06 \mathrm{mg} \mathrm{L}^{-1}\right)$ reported for a $25 \mu \mathrm{L}$ L-WS HPLC injection of a CC-hydrazone mix [32]. The $\mathrm{VA} / \mathrm{FA}$ molecular weight ratio is $86 / 30=\sim 3$. We make no attempt to reconcile our MDL results with the [32] data. The HPLC-MS MDL for PFPH derivatives (of FA, AA, PA, BA, and VA in gas samples) ranging from $0.21 \mathrm{ppb}$ (FA) to $0.10 \mathrm{ppb}$ (VA) [17] may generally reflect EI ionization efficiencies. More specifically, the maximum and minimum sensitivities are observed by FA and VA, respectively. As such, it is apparent that HPLC system is favorable to maintain enhanced sensitivity for the lighter CCs relative to the heavier CCs (on a per unit-weight basis-a molar basis, it is essentially constant). The cause of such systematic differences in HPLCbased analysis has been explored in our recent study [20].

In contrast, an opposing trend is apparent with the results derived by the GC method. The GC-based calibration 
data consistently indicate that the magnitude of RF values increases fairly systematically with increasing MWs, although their sensitivities are generally lower than the reference compound, benzene. (N.B.: the $70 \mathrm{eV}$ EI ionization cross sections increase linearly with MW.) As a result, the RF's (and also MDL's in mass units) will be essentially constant, irrespective of MW assuming quantitative recovery. Hence, the RF's (i.e., slope of peak area versus analyte weight) behavior for the GC-MS and HPLC-UV methods observed in this study are readily explainable in terms of EI ionization cross sections (see below for explanation, Section 3.3, last paragraph) and molar extinction coefficients, respectively.

3.2. Basic Quality Assurance of HPLC and GC-MS Method. The results of our comparative study show that the performance of the two systems is highly contrasting in many respects. The observed contradictory trend in relative sensitivity between the two methods is in fact reflected further, if comparison is extended in terms of the basic quality assurance (QA) parameters like precision or detection limits. To obtain the analytical precision for the CC determinations, the relative standard error of the mean (RSE: \%) was assessed based on the triplicate analyses of each standard phase by each method (Table 5). Comparison of RSE values of each method suggests that distinctions in reproducibility can be made between standard phases rather than intercompound relationships.

To conduct CC measurements, one needs to initiate the sampling step in which analytes in ambient air are enriched on an appropriate sampling medium via sorption $[33,34]$, cryotrapping [33, 35], or derivatization [24, 36, 37]. The detectability of CC is then determined not simply by the sensitivity of a given instrument but by its interactive relationship with those sampling methodologies. The detectability of each compound was also assessed in terms of method detection limit (MDL). The MDL for each CC was calculated by referring to the guidelines in 40 CFR Part 136, Appendix B [38] as the product of the standard deviation of seven replicates and the Student's $t$-value at the $99 \%$ confidence level $(t=3.14$ at $6 \mathrm{df})$.

The present work's detection limits for both systems are shown in Table 5. Evaluation of DL values shows an interesting trend. For both methods, detectability is greatly distinguished so that the maximum sensitivity is attained by lighter (HPLC) and heavier CCs (GC) in full compliance with the relative properties discussed above. These observed trends are due in part to molar extinction coefficients and EI ionization cross-sections considerations, thus reflecting the physics of the detectors being used. Explanations for the observed difference between the two systems can be sought mainly from the coupling effect between sample treatment procedures and the instrumental detection system such as effective derivatization of lighter CCs for HPLC analysis and preference of heavier CCs for TD-GC analysis.

The HPLC method is generally based on the derivatization technique to form a stable product in the reaction between analytes and the derivatizing reagent (for example: 2,4-dinitrophenylhydrazine [39]). In contrast, the GC-based analysis can be simplified, if coupled with the thermal desorption (TD) technique, while the adoption of the initial reaction (e.g., with derivatization) can be a selectable option [21]. A 1992 review reports that solid sorbent sampling with Tenax followed by TD/GC-FID analysis yielded CC detection limits of $<0.1 \mathrm{ppb}$ for $3 \mathrm{~L}$ samples [23]. Later in 2008, detection limits in the range of 0.75 to $2.33 \mathrm{ng}(\sim 0.1 \mathrm{ppb}$ calculated for $3 \mathrm{~L}$ samples) were reported for AA, PA, BA, IA, and VA using cold trapping/TD/GC-FID analysis; calibration properties were observed to be moderately dependent on TD settings [33]. A potentially attractive method that could be used for near real time general VOC analysis at the pptv level is proton transfer reaction mass spectrometry (PTR-MS) [40]. For example, a detection limit of $78 \mathrm{pptv}$ for FA has been reported using a PTR-MS equipped with a $-40^{\circ} \mathrm{C}$ sampling cryo-dehumidifier (containing amorphous silicon), $250 \mathrm{sccm}$ air sampling rate, and $5 \mathrm{~s}$ data acquisition time on $\mathrm{m} / \mathrm{z} 31$ (sample volume consumed $\sim 21 \mathrm{~mL}$ ) [41].

3.3. Relative Recovery (RR) of CC Standards between Different Methods. To analyze CC in gaseous media by the GC or HPLC methods, the use of preconcentration technique [18] is often a requisite choice to handle large sample volumes to overcome the trace analyte abundances per given sample matrix. Thus, the reliability of each method is determined by the cumulative effects of experimental biases stemming from the initial sample collection stage to the final detection/data analysis stages. Considering that the quantitation of CCs in gaseous media (air) is commonly made against standard prepared in liquid phase, relative recovery (RR) between standard phases can be used as a critical variable to assess the reliability of each method. Hence, the experimental performance of the two major detection techniques for CCs can be evaluated based on direct evaluation of the RR between liquid and gas phase standards.

It is interesting to note that HPLC system exhibits generally enhanced reproducibility from liquid phase standards to reflect the relatively simple steps involved in the calibration of L-WS. In contrast, enhanced reproducibility of gaseous CC standard is also apparent in the GC analysis. To learn more about the possible bias stemming from the use of different phase standards, the difference in RF values for a given method can be assessed in terms of RR between liquid and gas phases. Hence, as a means to assess the RR for a given compound, the percent difference (PD) was computed by dividing the observed RF differences between the two standard phases with that of liquid phase (Table 4).

The resulting $\mathrm{PD}$ values derived for each method also seem to comply with the patterns seen from the relative sensitivity derived by the interactive relationship between standard phases and instrumental setups. In the case of HPLC, the PD values tend to increase with the increasing MWs of the CCs. As such, comparison of relative ordering in $\mathrm{RF}$ values and of PD values consistently suggests that the use of HPLC should be less reliable for the heavier CCs than the lighter ones. On the other hand, an opposing trend is evident from the GC-based calibration data, as the PD values tend to decrease with increasing MWs. As such, the overall results of our comparative analysis confirm a strong and consistent 
trend in the analysis of CC analysis for a given instrumental system.

Table 4 presents the molar RF's (MRF) [defined as RF * $\mathrm{MW} / 1,000,000]$ obtained using HPLC-UV analysis. For the L-WS, all analyzed CCs have similar MRF values ranging from $9.6\left(\mathrm{FA}, e=17.5 \times 10^{3}\right)$ to $11.2\left(\mathrm{PA}, e=20.1 \times 10^{3}\right)$ and the MRF correlates reasonably well with the literature molar extinction coefficient (e) for the carbonyl-DNPH derivatives. On the other hand, the MRF values for the G-WS showed a general decrease with increasing $\mathrm{MW}$ ranging from 11.1 (FA, $\mathrm{MW}=30)$ to $5.4(\mathrm{IA}, \mathrm{MW}=86)$ excluding VA $(\mathrm{MRF}=8.2$, $\mathrm{MW}=86)$. Also shown in Table 4 are the relative recovery factors (RRF) with respect to benzene set at $100 \%$ for TDGC-EI-MS analysis of L-WS and G-WS. Based on work done in our laboratory, the aromatics (e.g., benzene) can be safely assumed to have near quantitative RFs. The relative EI ionization cross sections $\left(s_{r}\right)$ with respect to $\mathrm{N}_{2}\left(s_{r}=1.00\right)$ can be estimated from molecular polarizability (a) as follows: for hydrocarbons $s_{r}=0.50 a-0.05$ and (b) for VOCs (other than hydrocarbons) $s_{r}=0.36 a+0.30$ [42]. The molecular polarizabilities (a) were calculated using an additivity scheme based on atomic polarizabilities [43]. The following $s_{r}$ values (in parentheses) were estimated for B (5.15), AA (1.92), PA (2.58), BA (3.24), and IA/VA (3.90).

From the ionization response factors $\left(=s_{r} / \mathrm{MW}\right)$, the RRFs in parentheses relative to $\mathrm{B}(=100 \%)$ were estimated for AA (66\%), PA (67\%), BA (68\%), and IA/VA (69\%). Hence, very evidently, the RRF values for AA by GC-MS are very poor at $2.4 \%$ and $0.56 \%$ for L-WS and G-WS, respectively. The GCMS RRFs do however improve significantly in going from AA to VA/IA, approaching the values estimated from $s_{r}$ considerations. For example for IA, the RRF values were $61.3 \%$ and $60.4 \%$ for L-WS and G-WS, respectively. These values are close to the theoretical value of $69 \%$, suggesting an RF of IA to be $88 \%$. For the interested reader, the reported RRs ranged from $14 \%$ to $213 \%$ for 31 different CCs $\left(\mathrm{C}_{1}-\mathrm{C}_{13}\right)$ using DNPH cartridge-RRLC-UV analysis using a $\mathrm{MeOH} / \mathrm{THF} / \mathrm{iPrOH} / \mathrm{H}_{2} \mathrm{O}$ HPLC mobile phase [44].

\section{Conclusion}

In this research, the basic characteristics of the two key experimental approaches available for carbonyl analysis, namely GC and HPLC were investigated against both liquid and gas phase standards. The experimental uncertainties in the quantitative analysis of CCs were then assessed by examining the compatibility of calibration results derivable by all four combinations between two experimental methods and two standard phases. Considering that the availability of gas phase standard is virtually not possible for all different volatile components in air, information concerning compatibility between different standard phases can be a critical component in validating the feasibility of liquid phase standard in the quantitative analysis of gas samples. Moreover, the reliability of a given instrumental method needs to be assessed thoroughly for the target compounds, if multiple instrumental methods are available for the analysis.

The overall results of our study confirm a strong consistency in the analytical properties of the GC and HPLC methods in the quantitation of CCs in air. If the feasibility of a given method is assessed in terms of relative recovery, the patterns contrast greatly between the two methods. Firstly, the HPLC method can yield the most reliable results for the lighter CCs like FA and AA; in contrast, the GC method is found to yield enhanced recoveries in the higher CCs like BA, IA, and VA. Although the calibration of PA can be made with the high coefficient of determination from all different coupled systems, it can suffer most significantly from the recovery. The overall results of our study thus confirm that the maximum reliability of CC analysis can be attained, if their analysis is made for a given species with the optimum coupling between the instrumental method and standard phases. Considering that there are limitations in the applicability of each method in the detection of CCs, one needs to put more effort to extend their applicability (e.g., the use of improved derivatization techniques, e.g., PFPH) to yield the data sets for the maximum number of CCs with the least bias.

\section{Conflict of Interests}

The authors declare that there is no conflict of interests regarding the publication of this paper.

\section{Acknowledgment}

This work was supported by a Grant from the National Research Foundation of Korea (NRF) funded by the Ministry of Education, Science and Technology (MEST) (no. 20090093848). All the experimental work reported herein was conducted at the authors' previous affiliation: Department of Environment \& Energy, Sejong University, Seoul, Korea 143747.

\section{References}

[1] P. Carlier, H. Hannachi, and G. Mouvier, "The chemistry of carbonyl compounds in the atmosphere-a review," Atmospheric Environment A, vol. 20, no. 11, pp. 2079-2099, 1986.

[2] S. F. Maria, L. M. Russell, M. K. Gilles, and S. C. B. Myneni, "Organic aerosol growth mechanisms and their climate-forcing implications," Science, vol. 306, no. 5703, pp. 1921-1924, 2004.

[3] J. Lathière, D. A. Hauglustaine, A. D. Friend, N. de NobletDucoudré, N. Viovy, and G. A. Folberth, "Impact of climate variability and land use changes on global biogenic volatile organic compound emissions," Atmospheric Chemistry and Physics, vol. 6, no. 8, pp. 2129-2146, 2006.

[4] Y. Ji, Y. Gao, G. Li, and T. An, “Theoretical study of the reaction mechanism and kinetics of low-molecular-weight atmospheric aldehydes $\left(\mathrm{C}_{1}-\mathrm{C}_{4}\right)$ with $\mathrm{NO}_{2}$," Atmospheric Environment, vol. 54 , pp. 288-295, 2012.

[5] M. Z. Jacobson, "Effects of ethanol (E85) versus gasoline vehicles on cancer and mortality in the United States," Environmental Science and Technology, vol. 41, no. 11, pp. 4150-4157, 2007.

[6] E. Kabir and K.-H. Kim, "An investigation on hazardous and odorous pollutant emission during cooking activities," Journal of Hazardous Materials, vol. 188, no. 1-3, pp. 443-454, 2011.

[7] D. X. Ho, K.-H. Kim, J. R. Sohn, Y. H. Oh, and J.-W. Ahn, "Emission rates of volatile organic compounds released from 
newly produced household furniture products using a largescale chamber testing method," TheScientificWorldJournal, vol. 11, pp. 1597-1622, 2011.

[8] K.-H. Kim, S. A. Jahan, and E. Kabir, "A review of diseases associated with household air pollution due to the use of biomass fuels," Journal of Hazardous Materials, vol. 192, no. 2, pp. 425-431, 2011.

[9] S. K. Pandey and K.-H. Kim, "An evaluation of volatile compounds released from containers commonly used in circulation of sports beverages," Ecotoxicology and Environmental Safety, vol. 74, no. 3, pp. 527-532, 2011.

[10] S. D. Richardson, "Water analysis: emerging contaminants and current issues," Analytical Chemistry, vol. 81, no. 20, pp. 86548654, 2009.

[11] R. Pal, K.-H. Kim, Y.-J. Hong, and E.-C. Jeon, “The pollution status of atmospheric carbonyls in a highly industrialized area," Journal of Hazardous Materials, vol. 153, no. 3, pp. 1122-1135, 2008.

[12] M. Chai, M. Lu, F. Liang, A. Tzillah, N. Dendramis, and L. Watson, "The use of biodiesel blends on a non-road generator and its impacts on ozone formation potentials based on carbonyl emissions," Environmental Pollution, vol. 178, pp. 159-165, 2013.

[13] P. Dai, Y. Ge, Y. Lin, S. Su, and B. Liang, "Investigation on characteristics of exhaust and evaporative emissions from passenger cars fueled with gasoline/methanol blends," Fuel, vol. 113, pp.10$16,2013$.

[14] J. Yanowitz, K. Knoll, J. Kemper, J. Luecke, and R. L. McCormick, "Impact of Adaptation on flex-fuel vehicle emissions when fueled with E40," Environmental Science and Technology, vol. 47, pp. 2990-2997, 2013.

[15] J. J. Winebrake, J. J. Corbett, and P. E. Meyer, "Energy use and emissions from marine vessels: a total fuel life cycle approach," Journal of the Air and Waste Management Association, vol. 57, no. 1, pp. 102-110, 2007.

[16] R. Arvidsson, K. Fransson, M. Fröling, M. Svanström, and S. Molander, "Energy use indicators in energy and life cycle assessments of biofuels: review and recommendations," Journal of Cleaner Production, vol. 31, no. 3-4, pp. 54-61, 2012.

[17] X. Pang, A. C. Lewis, and J. F. Hamilton, "Determination of airborne carbonyls via pentafluorophenylhydrazine derivatisation by GC-MS and its comparison with HPLC method," Talanta, vol. 85, no. 1, pp. 406-414, 2011.

[18] M. Li, S. Biswas, M. H. Nantz, R. M. Higashi, and X.-A. Fu, "Preconcentration and analysis of trace volatile carbonyl compounds," Analytical Chemistry, vol. 84, no. 3, pp. 1288-1293, 2012.

[19] J. S. Herrington and M. D. Hays, "Concerns regarding 24-h sampling for formaldehyde, acetaldehyde, and acrolein using 2,4-dinitrophenylhydrazine (DNPH)-coated solid sorbents," Atmospheric Environment, vol. 55, pp. 179-184, 2012.

[20] S. K. Saha, S.-H. Jo, H.-N. Song, R. J. C. Brown, and K.-H. Kim, "Contrasting recovery patterns of 2, 4-dinitrophenylhydrazones (DNPH) derivative of carbonyls between liquid and gas phase standards using HPLC-based analysis," Atmospheric Environment, vol. 62, pp. 562-565, 2012.

[21] R. Pal and K.-H. Kim, "Experimental choices for the determination of carbonyl compounds in air," Journal of Separation Science, vol. 30, no. 16, pp. 2708-2718, 2007.

[22] R. Otson and P. Fellin, "A review of techniques for measurement of airborne aldehydes," Science of the Total Environment, vol. 77, no. 2-3, pp. 95-131, 1988.
[23] A. Vairavamurthy, J. M. Roberts, and L. Newman, "Methods for determination of low molecular weight carbonyl compounds in the atmosphere: a review," Atmospheric Environment A, vol. 26, no. 11, pp. 1965-1993, 1992.

[24] D. K. W. Wang and C. C. Austin, "Determination of complex mixtures of volatile organic compounds in ambient air: an overview," Analytical and Bioanalytical Chemistry, vol. 386, no. 4, pp. 1089-1098, 2006.

[25] A. Kumar and I. Víden, "Volatile organic compounds: sampling methods and their worldwide profile in ambient air," Environmental Monitoring and Assessment, vol. 131, no. 1-3, pp. 301-321, 2007.

[26] R. Barro, J. Regueiro, M. Llompart, and C. Garcia-Jares, "Analysis of industrial contaminants in indoor air-part 1. Volatile organic compounds, carbonyl compounds, polycyclic aromatic hydrocarbons and polychlorinated biphenyls," Journal of Chromatography A, vol. 1216, no. 3, pp. 540-566, 2009.

[27] M. Vogel, A. Büldt, and U. Karst, "Hydrazine reagents as derivatizing agents in environmental analysis - A critical review," Fresenius' Journal of Analytical Chemistry, vol. 366, no. 8, pp. 781-791, 2000.

[28] R. Pal and K.-H. Kim, "The effect of cold trap adsorbents on the gas chromatographic analysis of ambient VOCs under Peltier cooling conditions," Journal of Separation Science, vol. 31, no. 67, pp. 1100-1109, 2008.

[29] Y.-H. Kim and K.-H. Kim, "Novel approach to test the relative recovery of liquid-phase standard in sorbent-tube analysis of gaseous volatile organic compounds," Analytical Chemistry, vol. 84, no. 9, pp. 4126-4139, 2012.

[30] K.-H. Kim, Y.-H. Kim, and R. J. C. Brown, "Conditions for the optimal analysis of volatile organic compounds in air with sorbent tube sampling and liquid standard calibration: demonstration of solvent effect," Analytical and Bioanalytical Chemistry, vol. 405, pp. 8397-8408, 2013.

[31] W. Pötter and U. Karst, "Identification of Chemical Interferences in Aldehyde and Ketone Determination Using DualWavelength Detection," Analytical Chemistry, vol. 68, no. 19, pp. 3354-3358, 1996.

[32] J. H. Murillo, J. F. R. Marín, and S. R. Román, “Determination of carbonyls and their sources in three sites of the metropolitan area of Costa Rica, Central America," Environmental Monitoring and Assessment, vol. 184, no. 1, pp. 53-61, 2012.

[33] R. Pal and K.-H. Kim, "Gas chromatographic approach for the determination of carbonyl compounds in ambient air," Microchemical Journal, vol. 90, no. 2, pp. 147-158, 2008.

[34] B. Buszewski, T. Ligor, W. Filipiak, M. T. Vasconcelos, M. Pompe, and M. Veber, "Study of sorptive properties of trap systems for selective enrichment of volatile organic compounds from tobacco smoke samples," Toxicological and Environmental Chemistry, vol. 90, no. 1, pp. 51-64, 2008.

[35] A. Levart and M. Veber, "Determination of aldehydes and ketones in air samples using cryotrapping sampling," Chemosphere, vol. 44, no. 4, pp. 701-708, 2001.

[36] E. Woolfenden, "Sorbent-based sampling methods for volatile and semi-volatile organic compounds in air-part 2. Sorbent selection and other aspects of optimizing air monitoring methods," Journal of Chromatography A, vol. 1217, no. 16, pp. 26852694, 2010.

[37] E. Woolfenden, "Sorbent-based sampling methods for volatile and semi-volatile organic compounds in air-part 1: sorbentbased air monitoring options," Journal of Chromatography A, vol. 1217, no. 16, pp. 2674-2684, 2010. 
[38] USEPA, "Definition and Procedure for the Determination of the Method Detection Limit - Revision 1.11, Code of Federal Regulations, Title 40, Part 136, Appendix B, 1984. Fed. Regist. 1986, 51, 23703," 1986.

[39] H. A. Iddles and C. E. Jackson, "Determination of carbonyl compounds by means of 2,4-dinitrophenylhydrazine," Industrial and Engineering Chemistry, vol. 6, no. 6, pp. 454-456, 1934.

[40] W. Lindinger, A. Hansel, and A. Jordan, "On-line monitoring of volatile organic compounds at pptv levels by means of Proton-Transfer-Reaction Mass Spectrometry (PTR-MS) Medical applications, food control and environmental research," International Journal of Mass Spectrometry and Ion Processes, vol. 173, no. 3, pp. 191-241, 1998.

[41] B. T. Jobson and J. K. McCoskey, "Sample drying to improve HCHO measurements by PTR-MS instruments: laboratory and field measurements," Atmospheric Chemistry and Physics, vol. 9, pp. 19845-19877, 2009.

[42] J. E. Bartmess and R. M. Georgiadis, "Empirical methods for determination of ionization gauge relative sensitivities for different gases," Vacuum, vol. 33, no. 3, pp. 149-153, 1983.

[43] K. J. Miller and J. A. Savchik, "A new empirical method to calculate average molecular polarizabilities," Journal of the American Chemical Society, vol. 101, no. 24, pp. 7206-7213, 1979.

[44] S. D. M. Ochs, F. C. Albuquerque, M. C. G. P. Massa, and A. D. Pereira Netto, "Evaluation of $\mathrm{C}_{1}-\mathrm{C}_{13}$ carbonyl compounds by RRLC-UV in the atmosphere of Niterói City, Brazil," Atmospheric Environment, vol. 45, no. 29, pp. 5183-5190, 2011. 

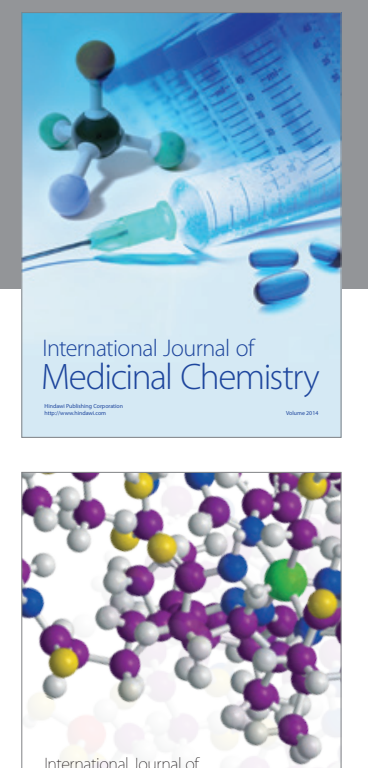

\section{Carbohydrate} Chemistry

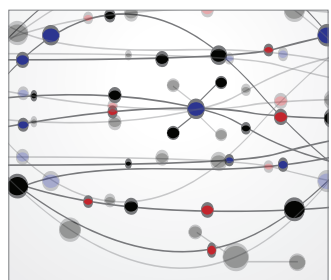

The Scientific World Journal
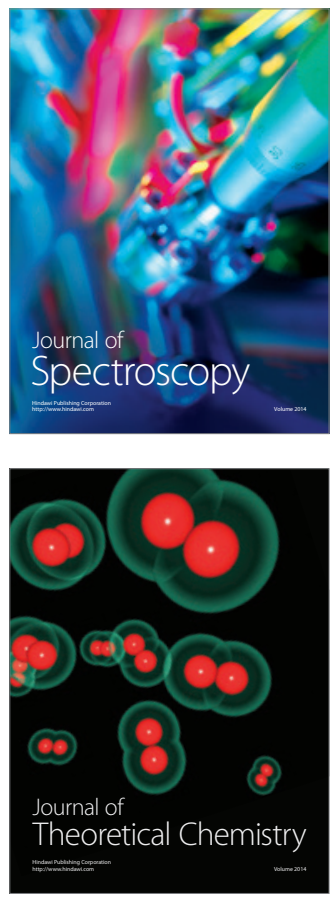
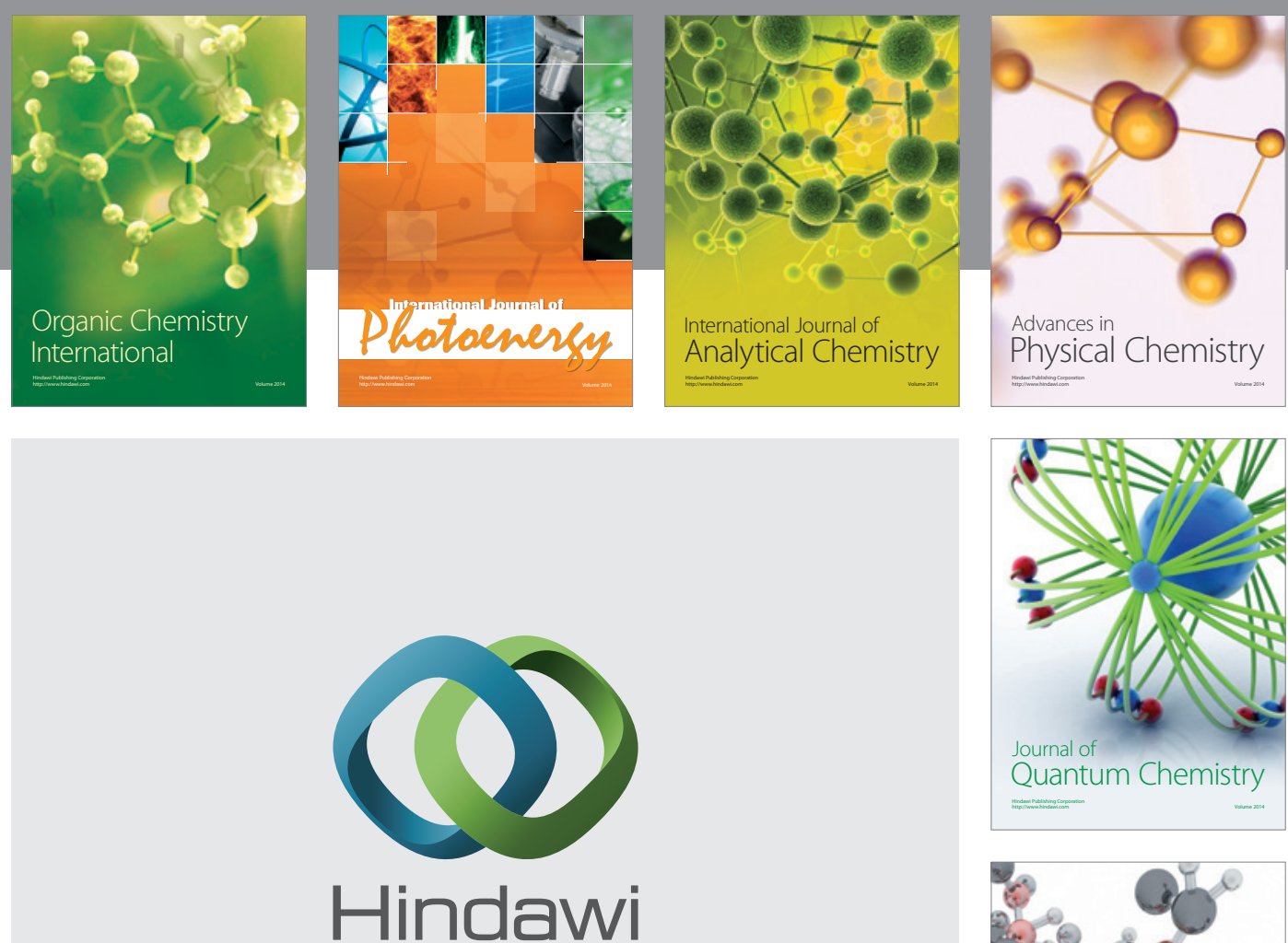

Submit your manuscripts at

http://www.hindawi.com

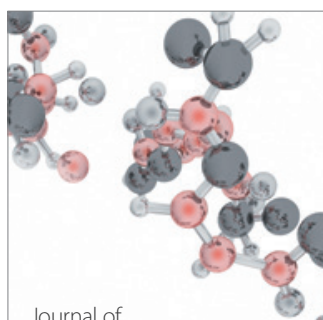

Analytical Methods

in Chemistry

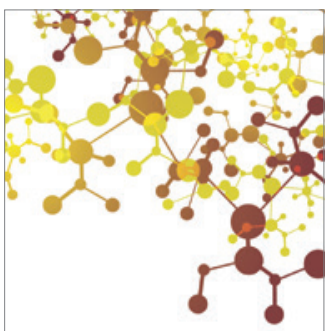

Journal of

Applied Chemistry

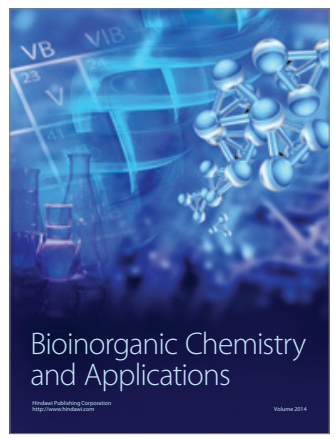

Inorganic Chemistry
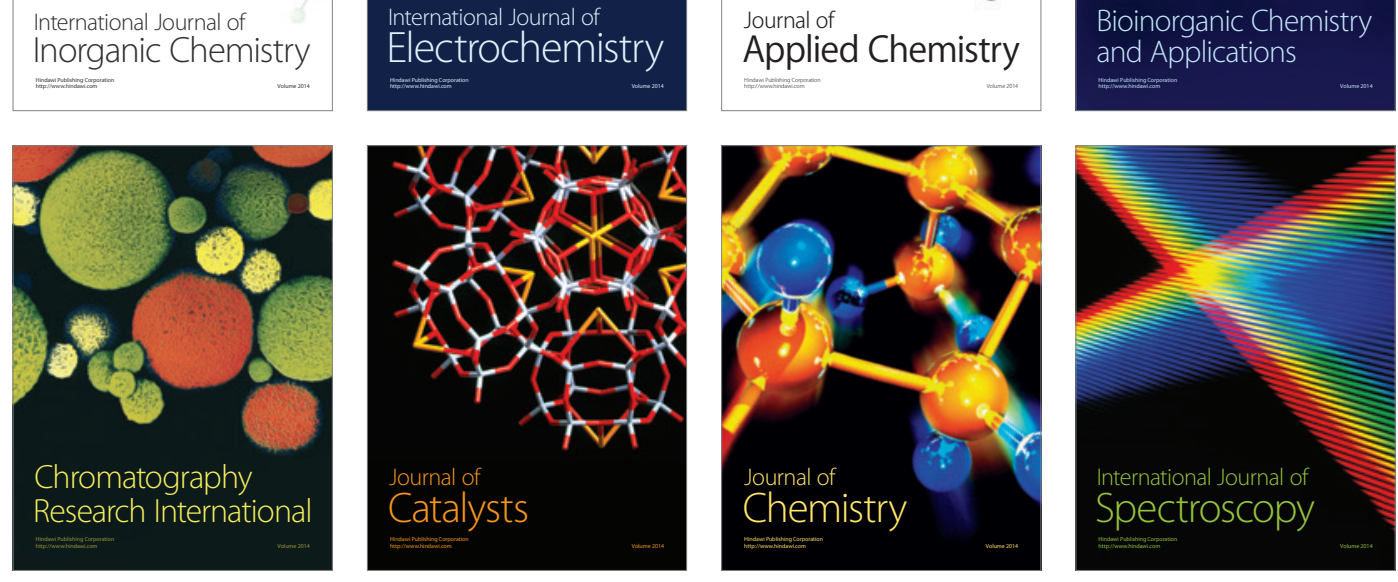